\title{
Tool Wear Detection Using Lipschitz Exponent and Harmonic Wavelet
}

\author{
Song Wanqing, Li Qing, and Wang Yuming \\ College of Electronic and Electrical Engineering, Shanghai University of Engineering and Science, Shanghai 201620, China
}

Correspondence should be addressed to Song Wanqing; swqls@126.com

Received 20 July 2013; Accepted 31 August 2013

Academic Editor: Carlo Cattani

Copyright (C) 2013 Song Wanqing et al. This is an open access article distributed under the Creative Commons Attribution License, which permits unrestricted use, distribution, and reproduction in any medium, provided the original work is properly cited.

The paper researches a novel engineering application of Lipschitz exponent function and harmonic wavelet for detecting tool condition. Tool wear affects often the quality grade of products and is gradually formed during cutting process. Meanwhile, since cutting noise is very strong, we think tool wear belongs to detecting weak singularity signals in strong noise. It is difficult to obtain a reliable worn result by raw sampled data. We propose singularity analysis with harmonic wavelet for data processing and a new concept of Lipschitz exponent function. The method can be quantitative tool condition and make maintaining decision. Test result was validated with 27 kinds of cutting conditions with the sharp tool and the worn tool; 54 group data are sampled by acoustic emission (AE).

\section{Introduction}

In recent years, great achievements in tool wear monitoring have been made based on the advanced mathematical models [1-8], even though none of these methods was successful online monitoring due to the complex performance of the machining processes. How to be quantitative tool condition monitoring is very important for practical engineering applications. This is a signal process problem that detects weak singularity signals in strong noise. However, the classical signal process is Fourier theory which has four defects [911]: (1) suits stationary stochastic signal, this kind of signal does not almost exists in engineering; (2) for discrete Fourier, Fourier analysis is precise with time series long from zero to infinite, otherwise truncation error is made; (3) weak singularity signal, relatively strange signal, is omitted in FFT however, weak singularity signal often contains important fault information; (4) when signal frequency happen a sudden change with adjacent time intervals, Fourier method only obtains two frequencies, it cannot display the moment two frequency changes or occurs (i.e., the singularities) in frequency domain. In order to overcome the above four defects to improve the measuring precision of weak singular signal in strong noise, we propose the combination between Lipschitz exponent index and complex wavelet for the detection of quantitative tool wear.
Lipschitz functions appear almost everywhere in mathematics [12]. Lipschitz functions are an important class of strong variations originating from smooth deformations of corresponding nonsmooth function [13]; the local regularity is often measured by Lipschitz exponents (LE); the singular variations can be viewed as combinations of weak increasing and decreasing variations; it means the case of discontinuity occurence on smooth function. Since the paper [14] is published in 1992 to detect signal's singularities, which proposed that the instantaneous frequencies can be approximately identified from the modulus maxima of a real wavelet based on general maxima, which are the locations of the largest modulus along maxima lines through an extension of [14], the complex-wavelet modulus maxima can detect and characterize singularities $[15,16]$ and has taken excellent result for measuring weak singularities of signal. Harmonic wavelet is one kind of complex wavelet; it is very sensitive to singularity feature of the signal that contains weak faults [17-20] and satisfies multiwavelets frames with arbitrary integer dilation factor [21]. The paper combines the modulus maxima of using a harmonic wavelet, and Lipschitz functions can be quantitative tool condition during cutting process. By 27 kinds of cutting conditions and turning experiments on HL-32 NC turning center, 54 group data are sampled by acoustic emission (AE). We have demonstrated 

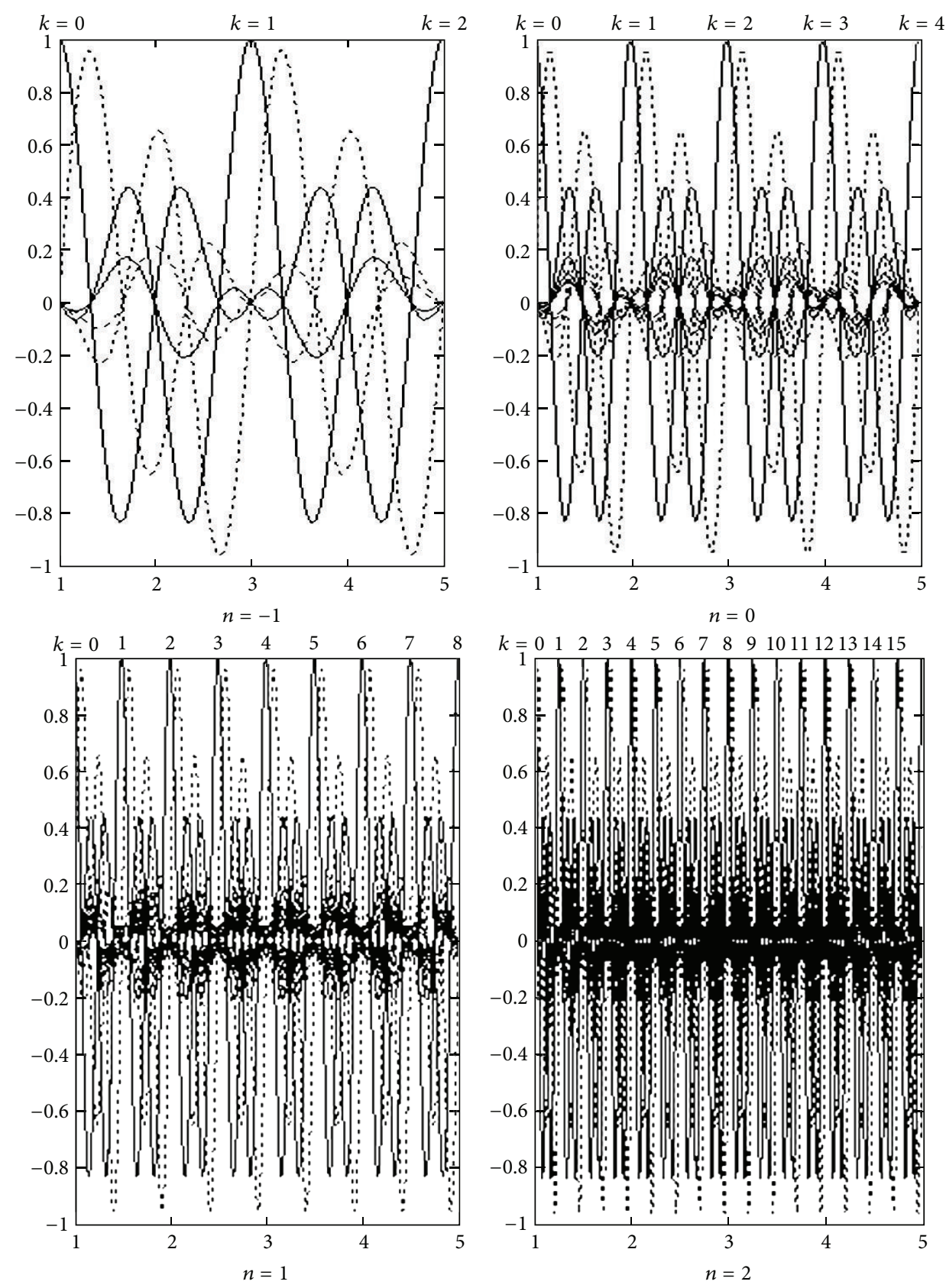

FIGURE 1: Real (thick line) and imaginary (thin line).

that combining the modulus maxima of harmonic wavelet and Lipschitz functions is better for detecting and estimating tool wear when a worn tool generates a singular signal in a strong noise.

\section{Harmonic Wavelet Transform Algorithm}

Discrete harmonic wavelets are given by

$$
\psi_{k}^{n}\left(2^{n} x-k\right) \stackrel{\operatorname{def}}{=} \frac{\exp \left(4 \pi i\left(2^{n} x-k\right)\right)-\exp \left(2 \pi i\left(2^{n} x-k\right)\right)}{2 \pi i\left(2^{n} x-k\right)},
$$

where $n=-1,0,1,2, \ldots, N . n$ is different levels (scale), and $k$ is different steps (time). The functions of its scale and time are given by Figure 1 .

The Fourier transform of (1) is as below:

$$
\left|\widehat{\psi}_{k}^{n}(\omega)\right|= \begin{cases}\frac{1}{2 \pi 2^{n}}, & 2 \pi 2^{n} \leq \omega \leq 2 \pi 2^{n+1}, \\ 0, & \text { other. }\end{cases}
$$

The amplitude-frequency characteristics of (2) is exactly like a box with band limited, as shown Figure 2. This characteristic is the best than any real wavelets for decomposed signal to difference frequency band. 


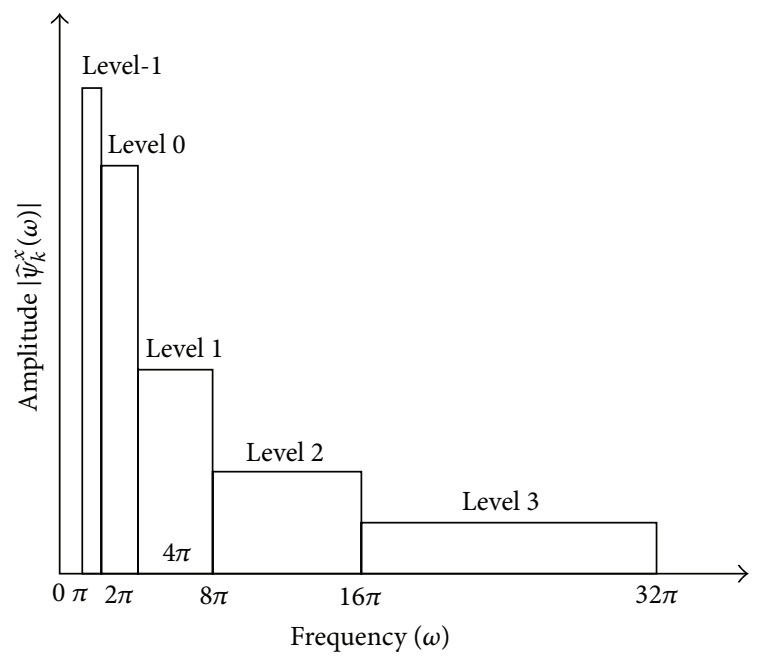

FIGURE 2: Amplitude-frequency characteristics of the discrete harmonic wavelets.

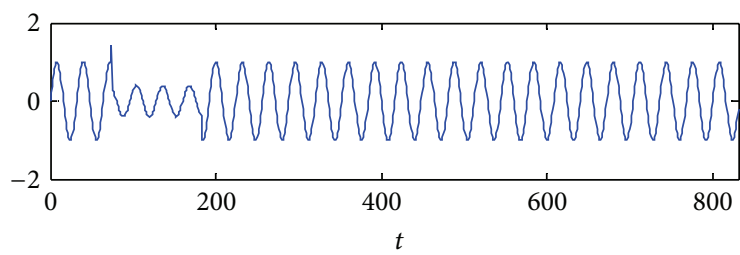

(a) Sine function with two singular points

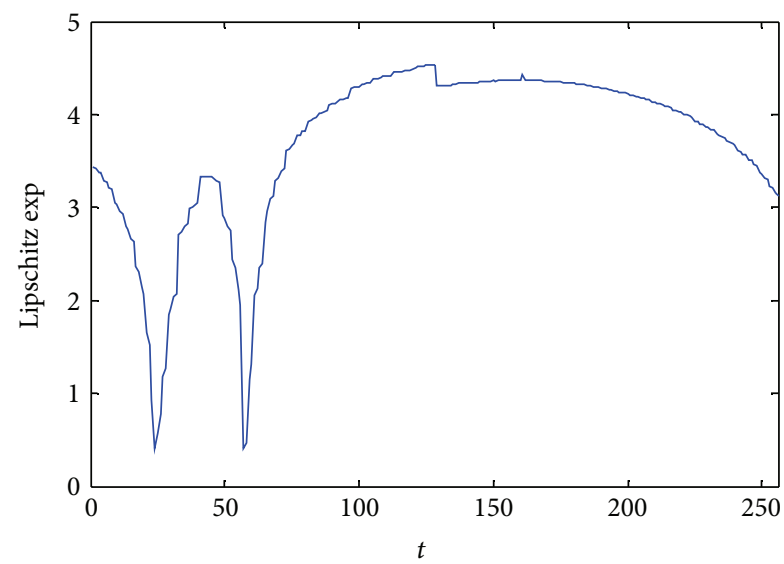

(b) $\alpha(t)$ along the temporal axis $t$

FIGURE 3: Extracting Lipschitz exponent function $\alpha(t)$.

\section{The Definition of Lipschitz Exponent}

Lipschitz is from Taylor formula $[16,22,23]$ Suppose that function $f(x)$ is $m$ times differentiable in $\left[x_{0}-h, x_{0}+h\right]$; then, Taylor expands in the neighborhood of $x_{0}$ :

$$
f_{x_{0}}(x)=\sum_{k=0}^{m-1} \frac{f^{(k)}\left(x_{0}\right)}{k !}\left(x-x_{0}\right)^{k}
$$

exists one approximate error:

$$
e(x)=f(x)-f_{x_{0}}(x)
$$

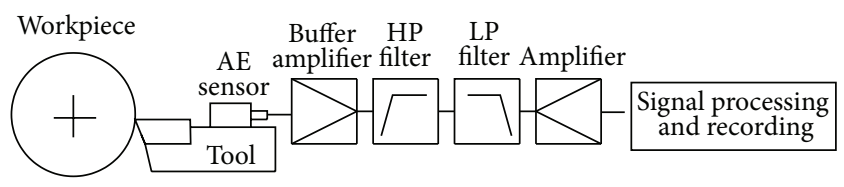

FIgURE 4: AE measurement in metal cutting.

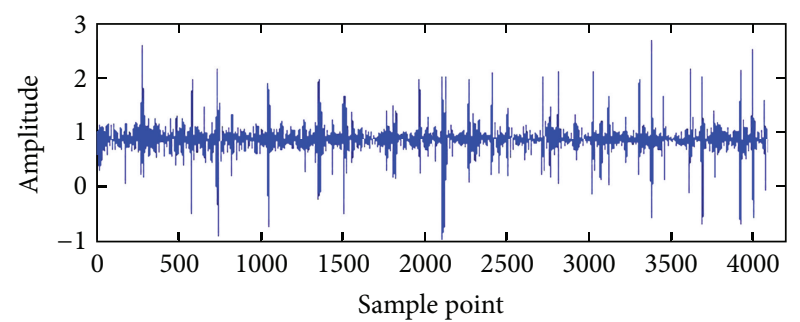

FIgURE 5: Waveform of sharp tool in first condition.

which satisfies:

$$
\begin{aligned}
& \forall x \in\left[x_{0}-h, x_{0}+h\right], \\
& |e(x)| \leq \frac{\left|x-x_{0}\right|^{m}}{m !} \sup _{\zeta \in\left[x_{0}-h, x_{0}+h\right]}\left|f^{m}(\zeta)\right| .
\end{aligned}
$$

The $m$ th order differentiability of $f(x)$ in the neighborhood of $x_{0}$ yields an upper bound on the error $e(x)$ when $x$ tends to $x_{0}$. The Lipschitz regularity refines this upper bound with noninteger exponents. The definition of Lipschitz exponent is as follows: a function $f(x)$ is of Lipschitz $\alpha$ at $x_{0}$, if and only if there exists a constant $A$ such that for all $x$ in a neighborhood of $x_{0}$

$$
\left|f(x)-f\left(x_{0}\right)\right| \leq A\left|x-x_{0}\right|^{\alpha} .
$$

We say that $f(x)$ is uniformly Lipschitz $\alpha$ for all $x$. Equation (6) is a kind of fractional order [20]. In a neighborhood of $x_{0}, f(x)$ is singular when $\alpha \neq 1$, and $f(x)$ is continuous when $\alpha>0$. In other words, If $f(x)$ is discontinuous and bounded in a neighborhood of $x_{0}$, then $\alpha=0$. If $f(x)$ is continuously differentiable then $\alpha=1$ and $f(x)$ is not singular.

\section{Modulus Maxima of Wavelet}

Reference [14] depicts the modulus maxima of real wavelet to detect singular signal, and [15] extends to the modulus maxima of complex wavelet, and algorithm is deduced in detail. We say a function $f(x)$ is a modulus maximum of wavelet at the point $\left(2^{n}, x_{0}\right)$; then,

$$
\left|\psi f\left(2^{n}, x\right)\right|<\left|\psi f\left(2^{n}, x_{0}\right)\right|
$$

or

$$
\frac{\partial \psi f\left(2^{j}, x_{0}\right)}{\partial x}=0,
$$

where $x$ belongs to either the right or the left neighborhood of $x_{0}$. A maxima line is consisted of a connected curve of the 
TABLE 1: Experimental cutting conditions.

\begin{tabular}{|c|c|c|c|c|c|c|c|}
\hline No. & $\begin{array}{l}\text { Speed } \\
\mathrm{r} / \mathrm{min}\end{array}$ & $\begin{array}{c}\text { Depth of cut } \\
\mathrm{mm}\end{array}$ & $\begin{array}{l}\text { Feed } \\
\mathrm{mm} / \mathrm{r}\end{array}$ & No. & $\begin{array}{l}\text { Speed } \\
\mathrm{r} / \mathrm{min}\end{array}$ & $\begin{array}{c}\text { Depth of cut } \\
\mathrm{mm}\end{array}$ & $\begin{array}{l}\text { Feed } \\
\mathrm{mm} / \mathrm{r}\end{array}$ \\
\hline 1 & 1500 & 1 & 0.1 & 15 & 1000 & 0.2 & 0.05 \\
\hline 2 & 1500 & 0.5 & 0.1 & 16 & 800 & 1 & 0.05 \\
\hline 3 & 1500 & 0.2 & 0.1 & 17 & 800 & 0.5 & 0.05 \\
\hline 4 & 1000 & 1 & 0.1 & 18 & 800 & 0.2 & 0.05 \\
\hline 5 & 1000 & 0.5 & 0.1 & 19 & 1500 & 1 & 0.02 \\
\hline 6 & 1000 & 0.2 & 0.1 & 20 & 1500 & 0.5 & 0.02 \\
\hline 7 & 800 & 1 & 0.1 & 21 & 1500 & 0.2 & 0.02 \\
\hline 8 & 800 & 0.5 & 0.1 & 22 & 1000 & 1 & 0.02 \\
\hline 9 & 800 & 0.2 & 0.1 & 23 & 1000 & 0.5 & 0.02 \\
\hline 10 & 1500 & 1 & 0.05 & 24 & 1000 & 0.2 & 0.02 \\
\hline 11 & 1500 & 0.5 & 0.05 & 25 & 800 & 1 & 0.02 \\
\hline 12 & 1500 & 0.2 & 0.05 & 26 & 800 & 0.5 & 0.02 \\
\hline 13 & 1000 & 1 & 0.05 & 27 & 800 & 0.2 & 0.02 \\
\hline 14 & 1000 & 0.5 & 0.05 & & & & \\
\hline
\end{tabular}

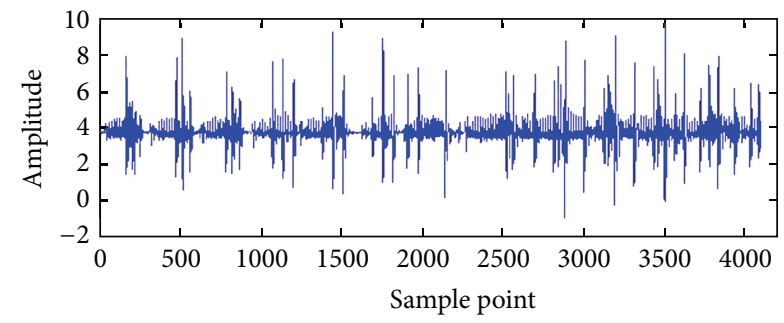

FIgURE 6: Waveform of wear tool in first condition.

modulus maxima in the scale space $n=-1,0,1,2, \ldots, N$. These multiscale modulus maxima are used to locate discontinuities [16], and if $\psi f\left(2^{n}, x\right)$ has no modulus maxima at fine scales, then $f(x)$ is locally regular. Singular point can be recognized by modulus maxima.

\section{Lipschitz Exponent Based on Modulus Maxima of Harmonic Wavelet}

It has been shown that the Lipschitz exponent of a local singularity can be characterized by being placed on their local modulus maxima at each scale $[14,16]$. References $[15,24]$ extend to using the modulus maxima of a complex wavelet. Here, we research that the modulus maxima of harmonic wavelet can be used to detect singularities, by means of a Lipschitz exponent of a function, and quantitative analysis on signal's singularities is measured. By examining the asymptotic decay of wavelet modulus maxima from coarser scale to finer scale, the strength of the singularity can be characterized by Lipschitz exponent.

Let $s=2^{n}, n=-1,0, \ldots, N$, in terms of $[15,25]$, function $f(x), x \in(a, b)$, and let $n$th $(n>0)$ derivative of $\operatorname{Re}(\psi f(s, x))$ and $\operatorname{Im}(\psi f(s, x))$ be finite deviations for each scale $s$. If a scale $s_{0}>0$ and constants $C$ and $A$ exist such that for $x \in(a, b)$ and $s>s_{0}$, all the modulus maxima of $\psi f(s, x)$ belong to a cone defined by

$$
\left|x-x_{0}\right| \leq C s
$$

such that at each modulus maxima $(s, x)$ in the cone

$$
|\psi f(s, x)| \leq A s^{\alpha}
$$

then $f(x)$ is uniformly Lipschitz $\alpha$ at $x$ when $x \neq x_{0}$, and $f(x)$ is Lipschitz $\alpha$ at $x_{0}$ when $\alpha<n$, and $\alpha$ is a noninteger. Equation (10) takes the logarithm on both sides:

$$
\log _{2}|\psi f(s, x)| \leq \log _{2} A+\alpha \log _{2} s .
$$

In $[15,24], A$ and $\alpha$ are computed by

$$
\min _{A, \alpha} \sum_{s}\left[\log _{2}|W f(s, x)|-\log _{2} A-\alpha * \log _{2} s\right]^{2}
$$

The problem of estimating $\alpha$ transforms into optimization based on a nonlinear least squared, and when (12) is minimized at all scale, $A$ and $\alpha$ are obtained.

According to [24] description of the results, we use $s=2^{n}$ instead of $s$, we get the following:

$$
\begin{aligned}
\alpha(x) & \\
= & \left(\sum_{n=-1}^{N}\left(\log _{2}\left|W f\left(2^{n}, x\right)\right| * \log _{2} 2^{n}\right)\right. \\
& \left.-\frac{\left(\sum_{n=-1}^{N} \log _{2}\left|W f\left(2^{n}, x\right)\right|\right)\left(\sum_{n=-1}^{N} \log _{2} 2^{n}\right)}{N}\right) \\
& \times\left(\sum_{n=-1}^{N}\left(\log _{2} 2^{n}\right)^{2}-\frac{\sum_{n=-1}^{N}\left(\log _{2} 2^{n}\right)^{2}}{N}\right)^{-1} .
\end{aligned}
$$



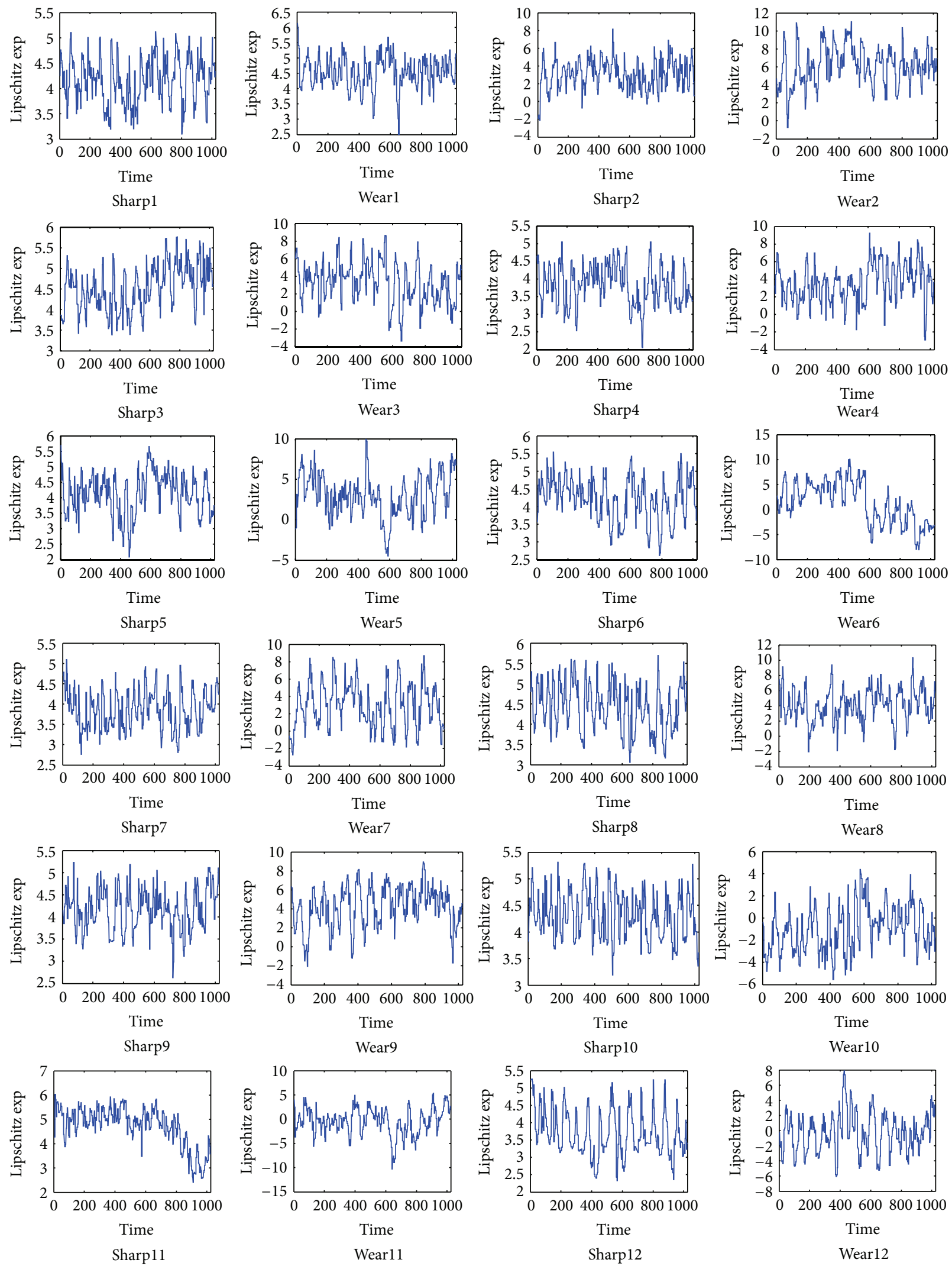

FIGURE 7: Continued. 

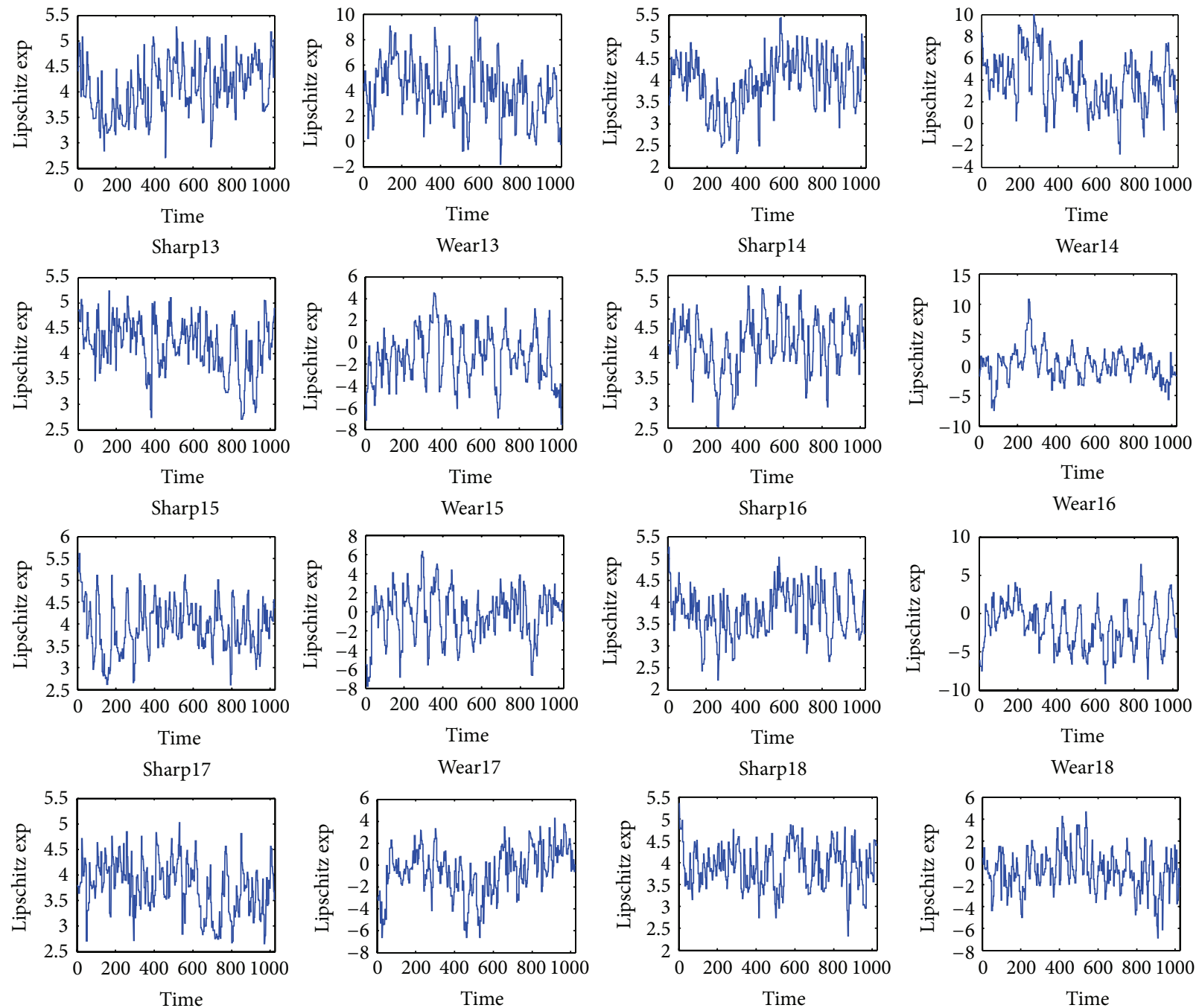

Sharp 18
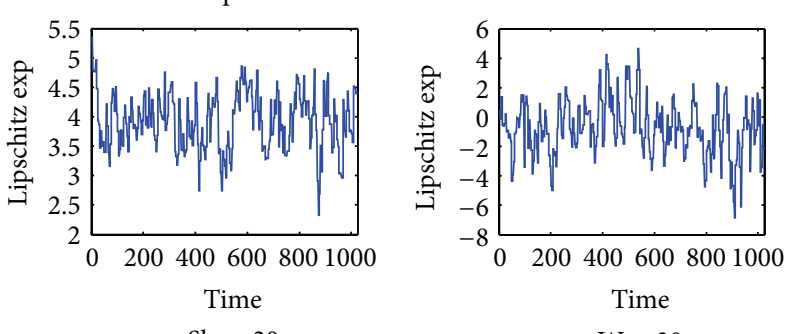

Sharp19
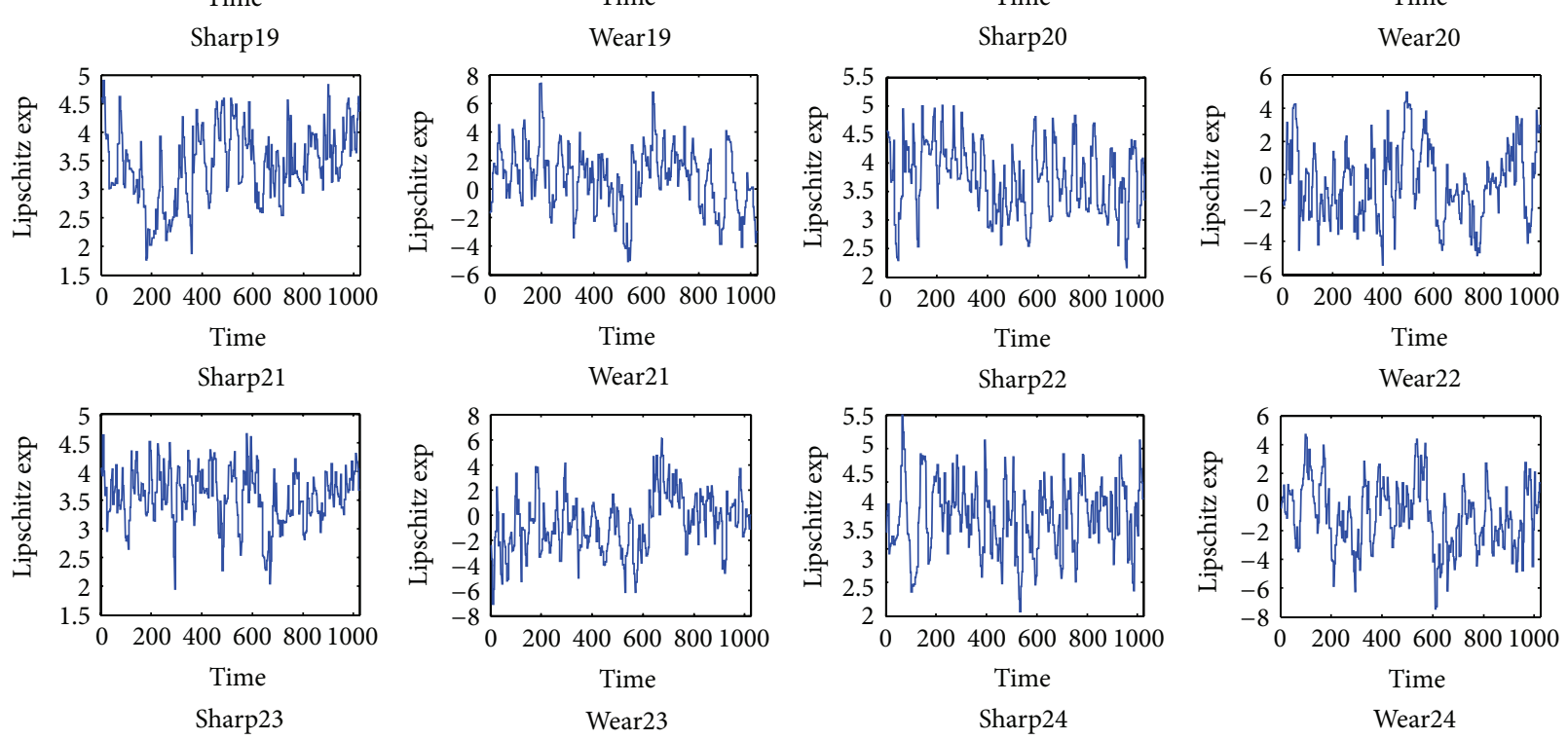

Figure 7: Continued. 

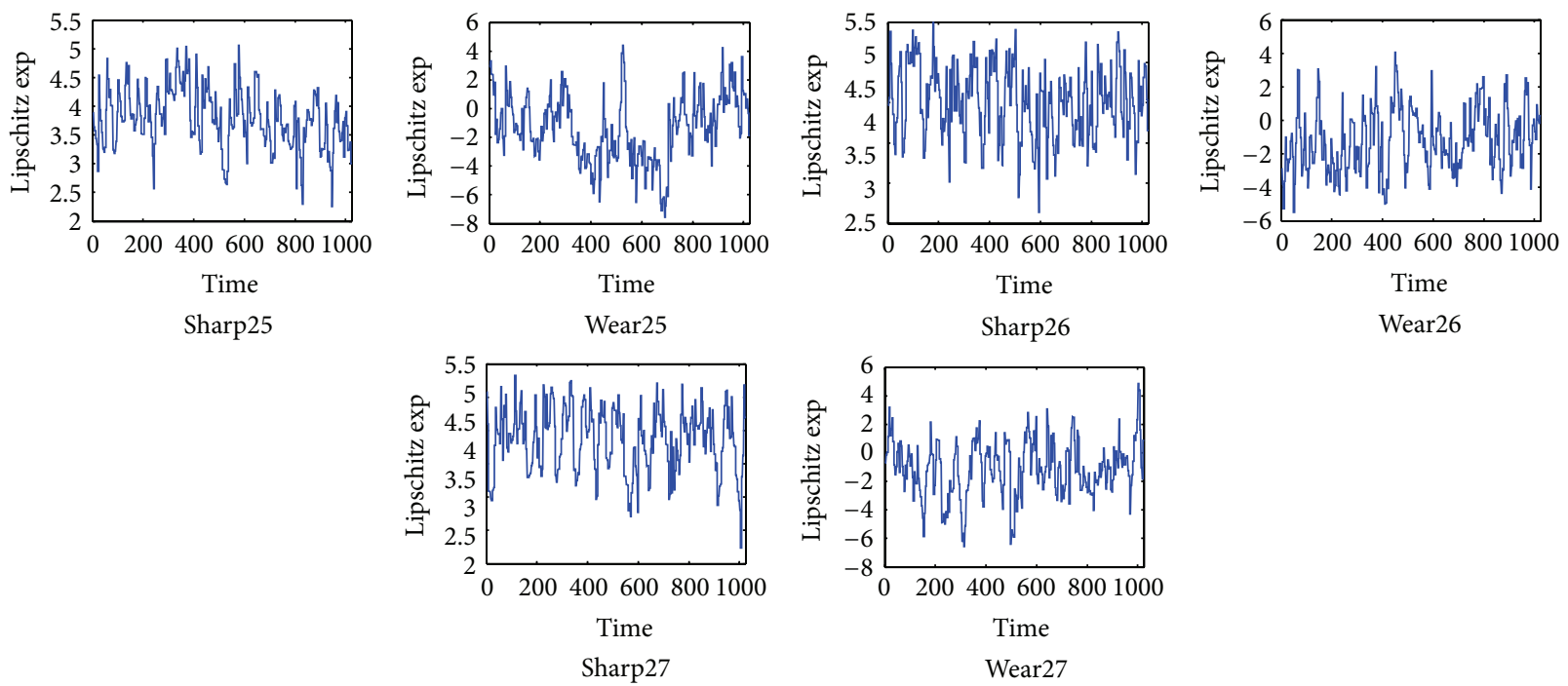

FIGURE 7: Lipschitz exponent function of 54 group data.

$\alpha(x)$ is called Lipschitz exponent function that describes the change of Lipschitz exponent value $\alpha$ along the temporal axis $x$. The variable $x$ is sampling point by acoustic emission (AE) during cutting process. Figure 5 is a simulation example of extracting Lipschitz exponent function $\alpha(x)$ on a sine function with singular points. Here, we set $N=10$ in (13).

Figure 3 demonstrates the potential ability of singularity detection (or fault detection) with Lipschitz exponent function $\alpha(x)$.

\section{Application Platform}

In turning experiments, the feasibility for tool condition monitoring is demonstrated by 27 kinds of cutting conditions (see Table 1); 54 group data are sampled by AE; they are shaper1 shaper27 and wear1 wear27, respectively. Machining tests were carried out on HL-32 NC turning center [26]. The work material was chosen for ease of machining, allowing for generation of surfaces of varying quality without the use of cutting fluids. The experiment equipment is shown in Figure 4. Currently, AE-based sensing technology is the area of most intense research activity for developing intelligent tool condition systems. The reason is that the sensitivity of AE to tool wear and fracture is coupled with a high response rate of the signal. Sharpl signal and wearl signal show Figures 5 and 6 , respectively in time domain.

According to (13), we set harmonic wavelet decomposed level $N=10$, and Lipschitz exponent function $\alpha(x)$ of 54 group data is shown in Figure 7.

In Figure 7, all sharp tools are of positive Lipschitz exponent function $\alpha(t)>0$, and all wear tools are of a negative Lipschitz exponent function $\alpha(t)<0$. Wear tool and sharp tool can be distinguished by Lipschitz exponent function $\alpha(t)$. A threshold value $\alpha(t)=0$ is obtained as a criterion of the tool condition monitoring during cutting process.

\section{Conclusion}

The proposed research provides a new theoretical basis and a new engineering application on the online tool monitoring which can distinguish between worn tool and sharp tool during cutting process. The results of experiment demonstrate that this method is more precise and robust.

The method which was described in this paper can be used as a valuable method for tool condition monitoring. In comparison to conventional data processing, the advantages of Lipschitz exponent and harmonic wavelet were shown. The zero threshold is determined conveniently which can distinguish between worn tool and sharp tool. For the future development of the presented techniques in laboratory, several approaches will be tested, including new broadband sensors application.

\section{Acknowledgment}

This project is supported by Shanghai Municipal Education Commission of China (Grant no. 13XKCZ07/YLJX12-7).

\section{References}

[1] V. B. Magdum and V. R. Naik, "Tool wear monitoring when turning EN 8 Steel with HSS-M2 tool," International Journal of Innovative Research in Science, Engineering and Technology, vol. 2, no. 5, pp. 1706-1711, 2013.

[2] Y. Huang and S. Y. Liang, "Modeling of cutting forces under hard turning conditions considering tool wear effect," Journal of Manufacturing Science and Engineering, Transactions of the ASME, vol. 127, no. 2, pp. 262-270, 2005.

[3] C.-W. Hung and M.-C. Lu, "Model development for tool wear effect on AE signal generation in micromilling," The International Journal of Advanced Manufacturing Technology, vol. 66, no. 9-12, pp. 845-1858, 2013. 
[4] M. A. Xavior and M. Adithan, "Determining the influence of cutting fluids on tool wear and surface roughness during turning of AISI 304 austenitic stainless steel," Journal of Materials Processing Technology, vol. 209, no. 2, pp. 900-909, 2009.

[5] R. Quiza, L. Figueira, and J. P. Davim, "Comparing statistical models and artificial neural networks on predicting the tool wear in hard machining D2 AISI steel," International Journal of Advanced Manufacturing Technology, vol. 37, no. 7-8, pp. 641648, 2008.

[6] S. Cho, S. Binsaeid, and S. Asfour, "Design of multisensor fusion-based tool condition monitoring system in end milling," International Journal of Advanced Manufacturing Technology, vol. 46, no. 5-8, pp. 681-694, 2010.

[7] D. Iliescu, D. Gehin, M. E. Gutierrez, and F. Girot, "Modeling and tool wear in drilling of CFRP," International Journal of Machine Tools and Manufacture, vol. 50, no. 2, pp. 204-213, 2010.

[8] F. Girardin, D. Rémond, and J.-F. Rigal, “Tool wear detection in milling-An original approach with a non-dedicated sensor," Mechanical Systems and Signal Processing, vol. 24, no. 6, pp. 1907-1920, 2010.

[9] A. E. Zonst, Understanding FFT Applications: A Tutorial for Laymen, Students, Technicians \& Working Engineers, Citrus Press, Titusville, Fla, USA, 2nd edition, 2003.

[10] J. F. James, A Student's Guide to Fourier Transforms: With Applications in Physics and Engineering, Cambridge University Press, London, UK, 3rd edition, 2011.

[11] A. H. Kaiser, Digital Signal Processing Using the Fast Fourier Transform (FFT), GRIN Verlag, Munich, Germany, 2013.

[12] J. Heinonen, "Lectures on Lipschitz Analysis, Lectures at the 14th Jyvaskyla Summer School in August 2004," Supported by NSF grant DMS 0353549 and DMS 0244421.

[13] Y. Grabovsky, V. A. Kucher, and L. Truskinovsky, "Weak variations of Lipschitz graphs and stability of phase boundaries," Continuum Mechanics and Thermodynamics, vol. 23, no. 2, pp. 87-123, 2011.

[14] S. Mallat and W. L. Hwang, "Singularity detection and processing with wavelets," IEEE Transactions on Information Theory, vol. 38 , no. 2, pp. 617-643, 1992.

[15] C.-L. Tu, W.-L. Hwang, and J. Ho, "Analysis of singularities from modulus maxima of complex wavelets," IEEE Transactions on Information Theory, vol. 51, no. 3, pp. 1049-1062, 2005.

[16] S. Mallat, A Wavelet Tour of Signal Processing, Academic Press, San Diego, Calif, USA, 3rd edition, 2008.

[17] C. Cattani, "Harmonic wavelet approximation of random, fractal and high frequency signals," Telecommunication Systems, vol. 43, no. 3-4, pp. 207-217, 2010.

[18] D. E. Newland, An Introduction to Random Vibrations, Spectral and Wavelet Analysis, Dover, London, UK, 3rd edition, 2005.

[19] D. E. Newland, "Harmonic wavelet analysis," Proceedings of the Royal Society of London. Series A, vol. 443, pp. 203-222, 1993.

[20] D. E. Newland, "Wavelet analysis of vibration, part 2: wavelet maps," Journal of Vibration and Acoustics, vol. 116, no. 4, pp. 417425, 1994.

[21] Y. Huang, Q. Li, and M. Li, "Minimum-energy multiwavelet frames with arbitrary integer dilation factor," Mathematical Problems in Engineering, vol. 2012, Article ID 640789, 37 pages, 2012.

[22] K. A. Innanen, "Local signal regularity and lipschitz exponents as a means to estimate Q, Journal of Seismic Exploration, vol. 12, no. 1, pp. 53-74, 2003.
[23] J. Lindenstrauss, D. Preiss, and J. Tiser, Fréchet Differentiability of Lipschitz Functions and Porous Sets in Banach Spaces (AM179), Princeton University Press, Princeton, NJ, USA.

[24] Q. Miao, H.-Z. Huang, and X. Fan, "Singularity detection in machinery health monitoring using Lipschitz exponent function," Journal of Mechanical Science and Technology, vol. 21, no. 5, pp. 737-744, 2007.

[25] P. Venkatakrishnan, S. Sangeetha, and M. Sundar, "Measurement of Lipschitz exponent (LE) using wavelet transform modulus maxima (WTMM)," International Journal of Scientific \& Engineering Research, vol. 3, no. 6, 2012.

[26] W. Song and J. Zhang, "Tool state detection by harmonic wavelet and sample entropy," Chinese Journal of Mechanical Engineering, vol. 24, no. 6, pp. 1068-1073, 2011. 


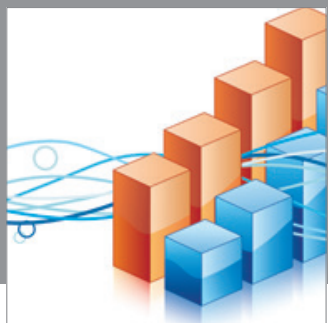

Advances in

Operations Research

mansans

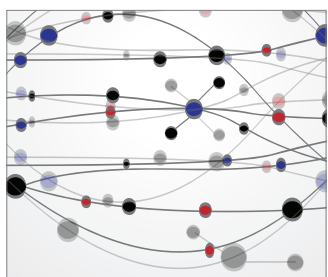

The Scientific World Journal
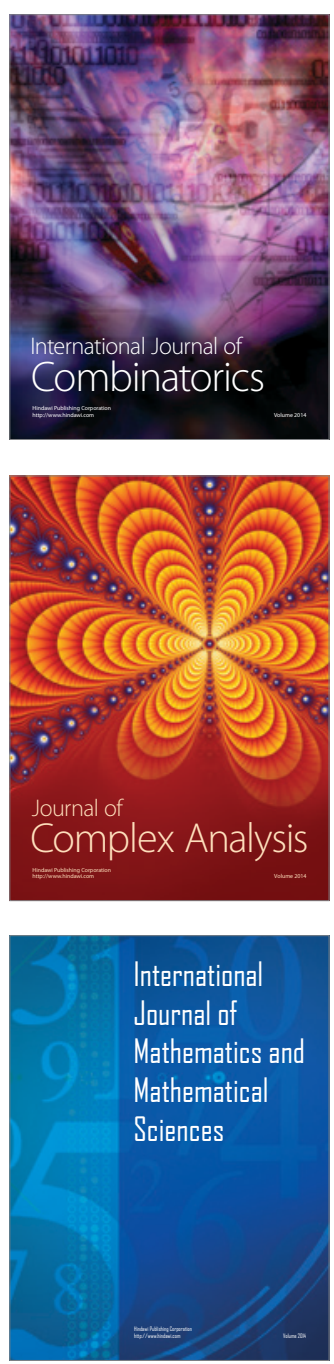
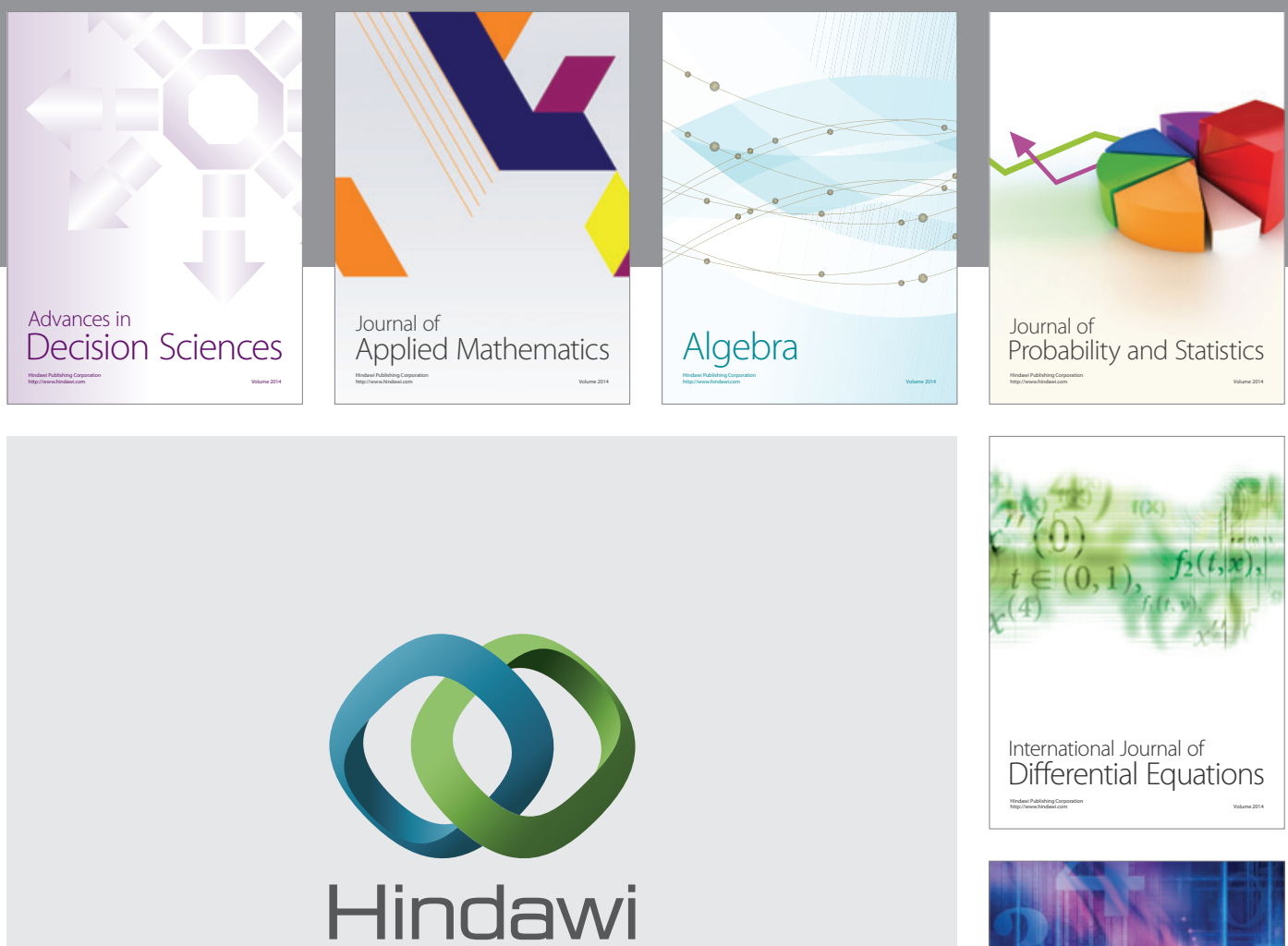

Submit your manuscripts at http://www.hindawi.com
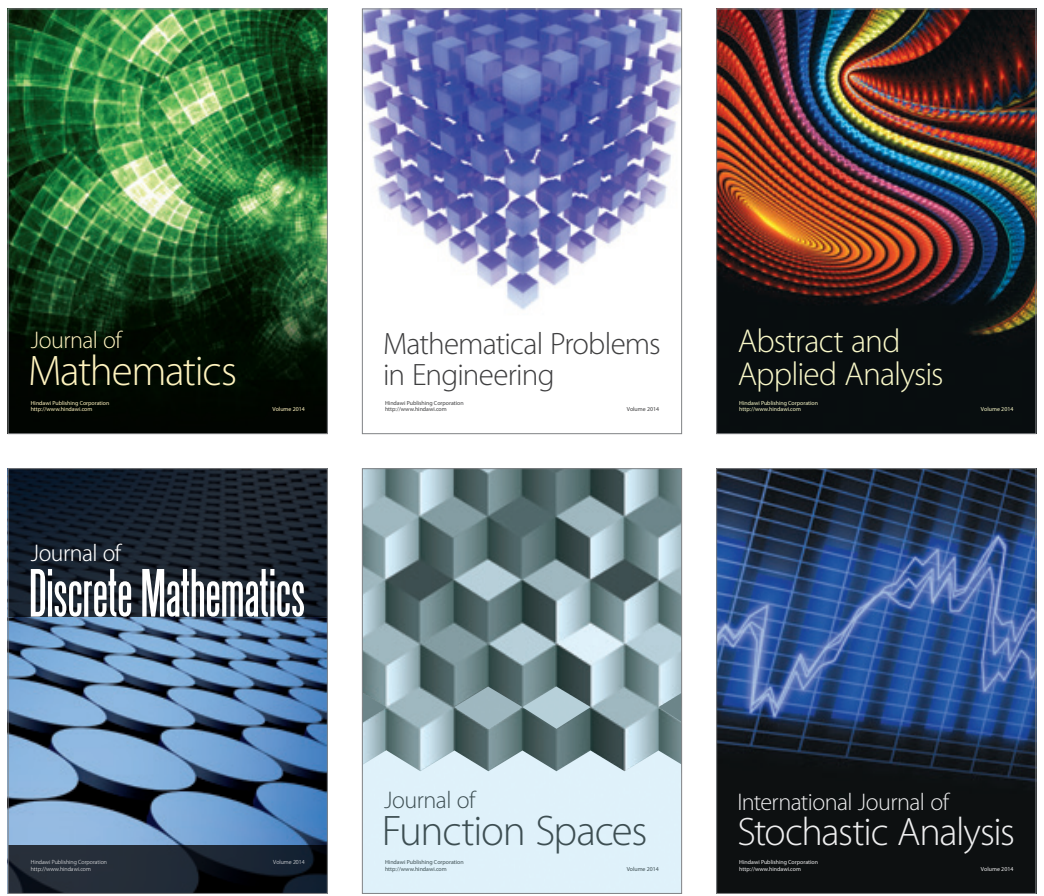

Journal of

Function Spaces

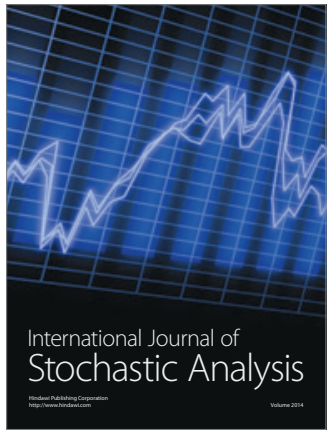

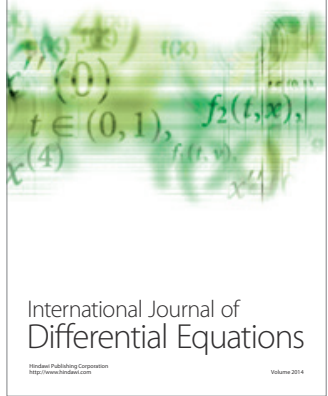
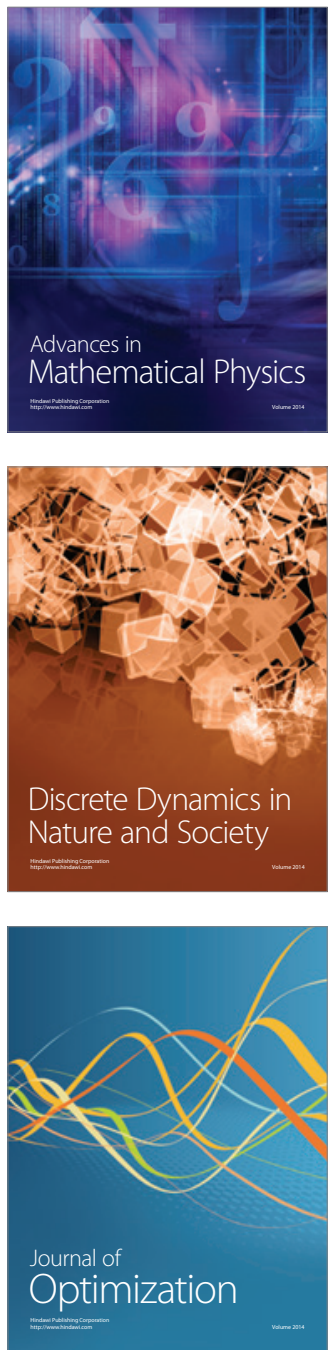